\section{Clinical features and natural history of acquired third, fourth, and sixth cranial nerve palsy}

\author{
U-C Park ${ }^{1,2}$, S-J Kim ${ }^{1,2}$, J-M Hwang ${ }^{1,2,3}$ and YS Yu ${ }^{1,2}$
}

\section{Introduction}

The third, fourth, and sixth cranial nerves participate in eyeball movement by extraocular muscle control. Acquired palsy of these nerves can result from various causes such as trauma, vascular disease, intracranial tumours, or aneurysm, and so on. The largest report from the Mayo Clinic, which included 4278 patients over 30 years, identified sixth nerve palsy and undetermined cause as having the highest prevalence. ${ }^{1}$ However, other studies showed variety in the aetiology and affected nerve distribution and wide range of recovery rates. ${ }^{1-6}$ The recovery from cranial nerve palsy is thought to have to do with its aetiology, but there have been rare attempts to identify the factors affecting recovery. ${ }^{1,4-6}$ We therefore performed this retrospective study to analyse the clinical features and natural history of acquired third, fourth, and sixth cranial nerve palsy and identify the factors affecting its recovery.

\section{Patients and methods}

All outpatients who directly visited or were referred from local ophthalmic practitioners to the neuro-ophthalmic service or strabismus service at ophthalmology department of Seoul National University Hospital for diplopia, blepharoptosis, or ocular motor restriction, and diagnosed with acquired third, fourth, and sixth cranial nerve palsy from July 1998 to February 2005 were included. Institutional Review Board Ethics Committee approval was obtained for this study, and it was carried out in adherence to the tenets of the Declaration of Helsinki. The patients' age of onset, sex, and previous medical history, especially the presence of vascular risk factors (diabetes, hypertension, ischaemic heart disease, vascular disease) were noted. The
${ }^{1}$ Department of Ophthalmology, Seoul National University College of Medicine, Seoul, Korea

${ }^{2}$ Seoul Artificial Eye Center, Seoul National University Hospital Clinical Research Institute, Seoul, Korea

${ }^{3}$ Department of Ophthalmology, Seoul National University Bundang Hospital, Seongnam, Korea

Correspondence: S-J Kim, Department of Ophthalmology, Seoul National University College of Medicine, \#28 Yongon-dong, Chongno-gu, Seoul 110744, Korea

Tel: 82-2-2072-2438;

Fax: 82-2-741-3187.

E-mail: ophjun@

snu.ac.kr

Received: 10 August 2006 Accepted in revised form: 12 December 2006 Published online: 9 February 2007 
degree of ocular motor restriction in the worst motion direction, the deviation angle at each visit, the presence of other related ophthalmic or neurological signs, and the results of neuroradiological tests were also recorded. Deviation angle was measured with the simultaneous prism and cover test in the primary position at distance fixation.

As in the Mayo Clinic series, cases were categorized into six aetiological groups according to the authors' best judgment: vascular, trauma, neoplasm, aneurysm, undetermined, or 'other' ${ }^{1-4}$ Vascular cases were defined as having at least one vascular risk factor (diabetes, hypertension, ischaemic heart disease, or peripheral vascular disease) without trauma history or evidence of aneurysm or neoplasm in image studies. 'Other', or miscellaneous, causes included complications of neurosurgical procedures and other systemic syndromes or diseases related to the cranial nerve function.

Congenital cases and other conditions that mimic cranial nerve palsy, such as orbital wall fracture, myasthenia gravis, and thyroid orbitopathy, were excluded. For the fourth cranial nerve palsy, history of head tilt dating back to infancy, definite facial asymmetry, amblyopia in the palsied eye, large vertical fusional amplitudes more than 10 prism dioptres (PD) were considered to be signs of congenital palsy and patients with any of these were excluded from the study.

The natural history of cranial nerve palsy was evaluated only in patients who received their first ocular exam within a month of palsy onset and followed for at least 6 months. We classified recovery at each follow-up visit as complete recovery, partial recovery, or persistence based on deviation angle and ocular motor restriction. Complete recovery was defined as the complete recovery of the angle of deviation, ocular motor restriction, and diplopia. Partial recovery was defined as a decrease in deviation angle by more than 10 PD from initial examination as measured in the primary position. For patients with an initial deviation angle of less than 10 PD, a decrease by more than $50 \%$ of the initial deviation angle was scored as partial recovery. Horizontal and vertical deviation angles in the primary position in fourth nerve palsy patients were much smaller than the general deviation angles of the third or sixth nerve palsy patients, even in the most severe cases. Therefore, in the fourth nerve palsy patient, a disappearance or decrease in the vertical deviation angle exaggerated with the head tilt test was used for the determination of recovery. Persistence was defined as the absence of total and partial recovery or cases that underwent extraocular muscle surgery. Baseline characteristics of the patients with complete recovery were compared to those without complete recovery by the $t$-test or $\chi^{2}$ test. Statistical analysis was performed with SPSS for Windows (version
12.0) and $P$-values less than 0.05 were considered significant.

\section{Results}

A total of 206 patients were included in the study and all of them were Koreans. The distributions of affected nerve, gender, laterality, aetiology, and average onset ages of each group are summarized in Table 1. For entire 206 patients, average onset age was 48.1 years (range, 1-83 years), and the vascular group had the highest onset age (63.9 years). The sixth nerve was most commonly affected $(n=108,52.4 \%)$ and the vascular disease was the most common aetiology $(n=64,31.1 \%)$.

The distribution of aetiologies within nerve groups showed similar distributions, except that there was no case of fourth nerve palsy caused by neoplasm or aneurysm, and that cases with 'other' aetiologies were more common in the sixth nerve palsy (20 of 108, 18.5\%) than in the third or fourth nerve palsy. Neoplasm as the cause of cranial nerve palsy included multiple neuromas in neurofibromatosis type 2 , brain stem glioma, juxtasellar meningioma, intracranial invasion of nasopharyngeal cancer, and posterior fossa epidermoid cyst. 'Other' causes included postoperative complications after neurosurgery, Miller-Fisher's syndrome, herpes zoster ophthalmicus, meningitis occurring after nerve block, mild subarachnoid haemorrhage, Tolosa-Hunt syndrome, and Wegener's granulomatosis.

A total of 108 patients had their first ocular exam within a month of palsy onset and followed for at least 6 months. Of these, 92 patients $(85.2 \%)$ showed at least partial recovery. Overall recovery (at least partial recovery) and complete recovery rates of each nerve group or aetiological group are shown in Table 2. Overall recovery rate was highest in patients with traumatic cause $(100.0 \%)$, followed by vascular cause $(86.7 \%)$ and undetermined cause $(86.2 \%)$.

Complete recovery was seen in 73 patients $(67.6 \%)$ and their average time interval from onset to complete recovery of the angle of deviation and ocular motor restriction was 3.5 months (range, 1-13 months). Further subgroup analysis for 151 patients who was first seen within 3 months of onset revealed overall recovery rate of $71.5 \%$ (108 of 151) and complete recovery rate of $55.6 \%$ (84 of 151).

Of seven patients who underwent treatments for intracranial mass or aneurysm, such as gamma knife surgery, radiotherapy, or aneurysm embolization with detachable coil, only one patient with internal carotid aneurysm showed reduction in size after embolization and the third nerve palsy partially recovered. Although remaining patients did not show mass size reduction 
Table 1 Distribution of the third, fourth, and sixth cranial nerve palsy patients by age, gender, laterality, and aetiology

\begin{tabular}{|c|c|c|c|c|c|c|c|}
\hline & & \multicolumn{4}{|c|}{ Nerve palsy } & \multirow[t]{2}{*}{ Mean age } & \multirow[t]{2}{*}{ Total } \\
\hline & & III & IV & $V I$ & Multiple & & \\
\hline $\mathrm{N}$ & & $48(23.3 \%)$ & $46(22.3 \%)$ & $108(52.4 \%)$ & $4(1.9 \%)$ & - & 206 \\
\hline Mean age & & $47.6 \pm 24.8$ & $50.8 \pm 16.9$ & $47.5 \pm 18.4$ & $40.5 \pm 17.2$ & $48.1 \pm 19.7$ & - \\
\hline Age range & & $1 \sim 77$ & $13 \sim 83$ & $3 \sim 83$ & $20 \sim 62$ & - & $1 \sim 83$ \\
\hline \multirow[t]{2}{*}{ Gender } & Male & 28 & 33 & 62 & 0 & $46.6 \pm 18.1$ & $123(59.7 \%)$ \\
\hline & Female & 20 & 13 & 46 & 4 & $50.4 \pm 21.8$ & $83(40.3 \%)$ \\
\hline \multirow[t]{3}{*}{ Laterality } & Right & 26 & 21 & 51 & 1 & - & $99(48.1 \%)$ \\
\hline & Left & 20 & 25 & 50 & 3 & - & $98(47.6 \%)$ \\
\hline & Bilateral & 2 & 0 & 7 & 0 & - & $9(4.4 \%)$ \\
\hline \multirow[t]{6}{*}{ Aetiology } & Vascular & 17 & 17 & 30 & 0 & $63.9 \pm 10.2$ & $64(31.1 \%)$ \\
\hline & Head trauma & 9 & 14 & 21 & 0 & $36.3 \pm 17.4$ & $44(21.4 \%)$ \\
\hline & Neoplasm & 3 & 0 & 6 & 0 & $33.6 \pm 19.8$ & $9(4.4 \%)$ \\
\hline & Aneurysm & 5 & 0 & 5 & 0 & $58.6 \pm 14.0$ & $10(4.9 \%)$ \\
\hline & Other & 5 & 2 & 20 & 4 & $36.6 \pm 19.4$ & $31(15.0 \%)$ \\
\hline & Undetermined & 9 & 13 & 26 & 0 & $45.8 \pm 17.5$ & $48(23.3 \%)$ \\
\hline
\end{tabular}

Table 2 Overall recovery (at least partial recovery), complete recovery rates, and initial deviation angle of each cranial nerve, and aetiological group for 108 patients, who had their first ocular exams within a month of palsy onset and at least 6-months follow-up

\begin{tabular}{llcccc}
\hline Group & Recovery/total & $\begin{array}{c}\text { Overall recovery } \\
\text { rate }^{\mathrm{b}}(\%)\end{array}$ & $\begin{array}{c}\text { Complete recovery } \\
\text { rate }(\%)\end{array}$ & $\begin{array}{c}\text { Initial deviation } \\
\text { angle }(\text { PD) }\end{array}$ \\
\hline Nerve & III & $16(13) / 19$ & 84.2 & 68.4 & $26.2 \pm 16.1$ \\
& IV & $19(12) / 23$ & 82.6 & 52.2 & $14.4 \pm 6.6$ \\
& VI & $55(46) / 63$ & 87.3 & 73.0 & $23.8 \pm 10.5$ \\
& Multiple & $2(2) / 3$ & 66.7 & 66.7 & $38.3 \pm 16.1$ \\
Aetiology & Vascular & $39(28) / 45$ & & & \\
& Head trauma & $8(7) / 8$ & 86.7 & 62.2 & $19.8 \pm 9.7$ \\
& Neoplasm and aneurysm & $3(2) / 5$ & 100.0 & 40.0 & $19.3 \pm 6.3$ \\
& Other & $17(16) / 21$ & 80.0 & 76.2 & $27.0 \pm 15.2$ \\
\multirow{2}{*}{ Total } & Undetermined & $25(20) / 29$ & 86.0 & $30.8 \pm 13.0$ \\
& & $92(73) / 108$ & 85.2 & 69.0 & $21.3 \pm 13.2$ \\
\hline
\end{tabular}

Abbreviation: PD, Prism dioptre.

anumber in the parenthesis indicates patients who had complete recovery.

${ }^{\mathrm{b}}$ Overall recovery $=$ complete recovery + partial recovery.

confirmed by image study after treatment, two patients with intracranial aneurysm and brainstem glioma, having initial eyeball deviation of less than $20 \mathrm{PD}$, showed recovery during their follow-up period. Of the patients with 'other' causes, most cases caused by inflammatory or infectious disease showed recovery after control of primary disease process, in spite of severe initial eyeball deviation larger than 40 PD.

Pupillary involvement was found in 15 patients, 13 of whom had the third nerve palsy. At final visits, only one patient showed complete resolution of pupillary defect after treatment of Tolosa-Hunt syndrome. Ptosis was found in 29 of 48 third nerve palsy patient (60.4\%) and of the 15 patients who received first ocular exam within 3 months of onset, $12(80 \%)$ showed recovery of ptosis.
In comparison between the patients with and without complete recovery, no significant differences were found in the gender, average onset age, palsied nerve, aetiology and ocular motor restriction (Table 3). Only deviation angle on first visit showed significant difference $(P=0.007)$. Complete recovery pattern of 108 patients according to aetiology and deviation angle on the first visit is shown in the Figure 1 and patients with smaller deviation angle are more likely to completely recover across all the aetiological groups.

\section{Discussion}

Our data confirmed the palsied nerve distribution reported in the previous studies, which usually found the 
Table 3 Comparison between the patients with and without complete recovery for 108 patients who had their first ocular exams within a month of palsy onset and at least 6-months follow-up

\begin{tabular}{|c|c|c|c|c|}
\hline & & Complete recovery & $\begin{array}{l}\text { Partial recovery } \\
\text { and persistence }\end{array}$ & P-value \\
\hline \multirow[t]{2}{*}{ Sex } & Male & 44 & 16 & \multirow[t]{2}{*}{$0.214^{\mathrm{a}}$} \\
\hline & Female & 29 & 19 & \\
\hline Age of onset & & $56.5 \pm 18.2$ & $50.9 \pm 18.2$ & $0.135^{\mathrm{b}}$ \\
\hline \multirow[t]{3}{*}{ Nerve palsy } & III & 13 & 6 & \multirow[t]{3}{*}{$0.315^{c}$} \\
\hline & IV & 12 & 11 & \\
\hline & VI & 46 & 17 & \\
\hline \multirow[t]{5}{*}{ Aetiology } & Vascular & 28 & 17 & \multirow[t]{5}{*}{$0.371^{\mathrm{a}}$} \\
\hline & Head trauma & 7 & 1 & \\
\hline & neoplasm and aneurysm & 2 & 3 & \\
\hline & Other & 16 & 5 & \\
\hline & Undetermined & 20 & 9 & \\
\hline \multicolumn{5}{|l|}{ Deviation angle (PD) } \\
\hline On first visit & & $20.5 \pm 11.3$ & $27.1 \pm 12.5$ & $0.007^{\mathrm{b}}$ \\
\hline \multirow[t]{5}{*}{ Ocular motor restriction on first visit } & $-1 / 2$ & 8 & 2 & \multirow[t]{5}{*}{$0.115^{\mathrm{a}}$} \\
\hline & -1 & 25 & 9 & \\
\hline & -2 & 22 & 7 & \\
\hline & -3 & 12 & 8 & \\
\hline & -4 & 6 & 9 & \\
\hline
\end{tabular}

Abbreviation: PD, prism dioptre.

${ }^{\mathrm{a}} \chi^{2}$ test.

bt-test.

'Three cases of multiple cranial nerve palsy were not included in $\chi^{2}$ test.

sixth cranial nerve palsy as the most common, accounting for $40.0-57 \%$ of total cases and the fourth nerve palsy as the least frequent. ${ }^{1-6}$ However, onset age of 48.1 was somewhat younger than 52.3 or 62.5 years of two reports, which included the three types of cranial nerve palsy altogether and described the onset age in detail. ${ }^{5,6}$ In the previous studies, aetiology distributions were various: vascular, undetermined cause, and neoplasm have been reported to be the most common. ${ }^{1-8}$ The most common aetiology had changed even along the four successive reports from the same institute..$^{1-4}$

This variety of the aetiology distribution may be attributable to ambiguous inclusion criteria for vascular cause. Differential diagnosis between a vascular and an undetermined aetiology may be challenging, and is usually based on the presence of vascular risk factors. ${ }^{5}$ In studies that considered only hypertension, diabetes, and ischaemic heart disease as vascular risk factors, vascular aetiology made up only $10.7-15.4 \%$ of total cranial nerve palsy patients. ${ }^{1-4}$ However, $40 \%$ of patients were attributable to vascular aetiology in another study that adopted hyperlipidemia, smoking, findings of microangiopathy, or lacunar infarction on brain imaging as additional vascular risk factors. ${ }^{6}$ Despite the introduction of useful diagnostic tools such as MRI, undetermined cause still occupy $17.7-35 \%$ of total cranial nerve palsy patients even in the reports after $1990 .{ }^{1,5,6}$ In previous reports, recovery rates of patients with undetermined cause (51.0-84.0\%) were similar to those of patients with vascular cause (70.9-100\%) and greater than other aetiological groups, as is in our study. ${ }^{1,-6}$ This suggests that a large portion of the undetermined aetiology group might share a same cause with the vascular group, such a kind of microvasculopathy undetectable by laboratory and imaging tests. ${ }^{1,5}$

We evaluated recovery only in 108 patients with their first ocular exam within 1 month of onset, and at least 6 months of follow-up. Patients with longer latency to their first exam might have experienced recovery during the intervening period, resulting in bias toward a lower recovery rate. ${ }^{9}$ Lower recovery rate in another subgroup analysis for 151 patients seen within 3 months of onset supports this idea. This also suggests that recovery starts as early as within 1 month of onset and those who were first seen between 1 and 3 month of onset might have less latent potentiality for further recovery than those seen within a month.

Previous studies reported the cranial nerve palsy recovery rates of $38.0-80 \%{ }^{1,4-6,10}$ Wide ranges of recovery rates is thought to result from variable criteria for recovery among the studies and criteria that are often not well defined. The use of objective criteria like ours, based 


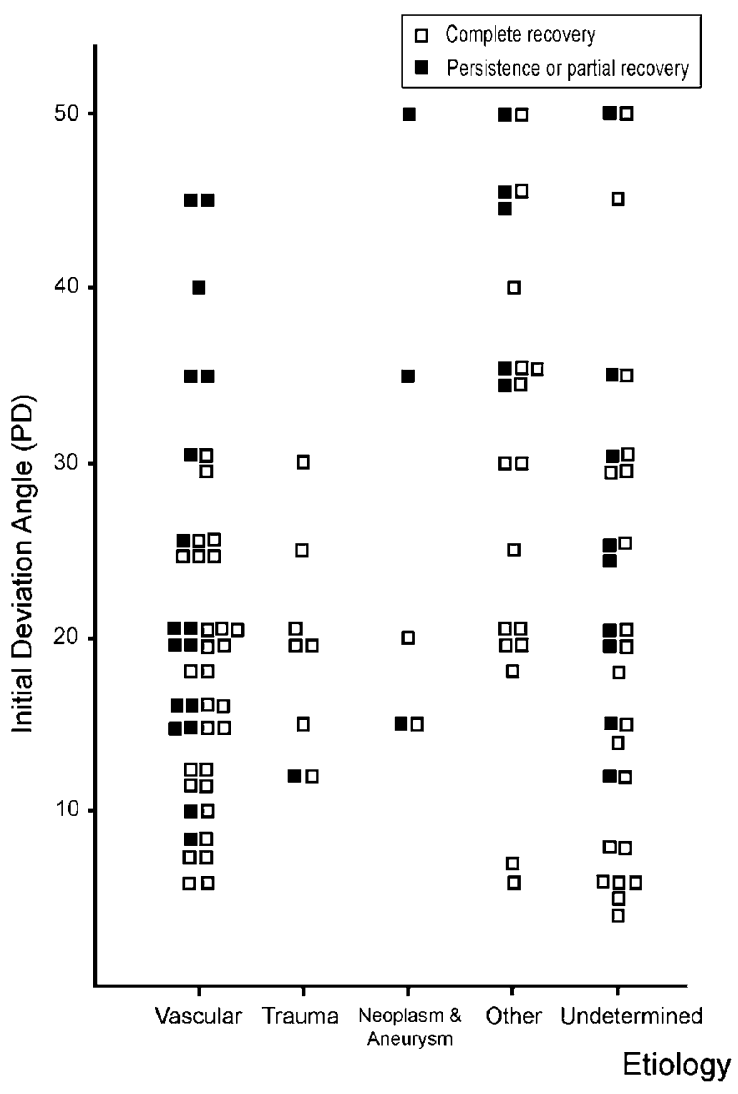

Figure 1 Complete recovery distribution of 108 patients who had their first ocular exams within a month of palsy onset and at least 6-month follow-up according to initial deviation angle and aetiology.

on eyeball deviation and motor restriction in patients who visited the clinic shortly after onset, may be helpful to obtain more consistent recovery rates. In some studies, recovery data were collected using follow-up letters or telephone calls in certain patients, not by ophthalmologic exam, but this might have led to inaccurate recovery rates. As to referral pattern, because most of patients first seen at local ophthalmologic clinics were referred to us as soon as possible for brain image study, referral practice pattern is not thought to have had effect on our recovery results.

Univariate analysis revealed that the deviation angle on the first visit was the only significant factor affecting recovery. Holmes et al ${ }_{1}^{11}$ in their study on treatment outcome of the sixth nerve palsy, also mentioned that patients who showed spontaneous recovery had smaller deviation angles, but they could not rigorously test their hypothesis because of small sample size. Although insignificant, vascular and undetermined groups showed higher overall recovery rate than other aetiological groups except trauma, as revealed in previous studies. ${ }^{1,-6}$ Overall recovery rate of $100 \%$ in trauma group might have resulted from that trauma patients seen within a month in this study had smaller deviation angle than others, as shown in Figure 1. However, aetiology is thought to have been confounding factors in previous studies. Our results show that vascular or undetermined groups have smaller initial eyeball deviation (Table 2). We postulate that lower recovery rates in grave aetiological groups in previous studies might have resulted from the larger initial deviation and motor restriction in these groups. Even patients with neoplasm recover well without treatment or mass size reduction if their initial deviations were small.

In addition to the initial deviation, successful management of treatable underlying disease is thought to be another factor affecting recovery from cranial nerve palsy. After size reduction of intracranial mass or aneurysm and after control of underlying inflammation and infection, severity of eyeball deviation, and motor restriction turned out to improve.

The results of this study are limited by its retrospective nature and relatively small sample size. However, this is the first study to evaluate the recovery of third, fourth, and sixth cranial nerve palsy with objective criteria based on deviation angle and to identify factors affecting recovery. With an attempt to minimize the bias toward a lower recovery rate, we observed overall recovery rate of $85.2 \%$ for the entire third, fourth, and sixth cranial nerve palsy. Our analysis suggests that deviation angle on first ocular examination and successful management of treatable underlying disease are important factors affecting recovery from cranial nerve palsy.

\section{Acknowledgements}

This study was supported by a grant of the Korea Health 21 R\&D Project, Ministry of Health \& Welfare, Republic of Korea (01-PJ1-PG1-01CH16-0002).

\section{References}

1 Richards BW, Jones FR, Younge BR. Causes and prognosis in 4278 cases of paralysis of the oculomotor, trochlear, and abducens cranial nerves. Am J Ophthalmol 1992; 113: 489-496.

2 Rucker CW. Paralysis of the third, fourth, and sixth cranial nerves. Am J Ophthalmol 1958; 46: 787-794.

3 Rucker CW. The causes of paralysis of the third, fourth, and sixth cranial nerves. Am J Ophthalmol 1966; 61: 1293-1298.

4 Rush JA, Younge BR. Paralysis of cranial nerves III, IV, and VI: cause and prognosis in 1000 cases. Arch Ophthalmol 1981; 99: 76-80.

5 Tiffin PA, MacEwen CJ, Craig EA, Clayton G. Acquired palsy of the oculomotor, trochlear and abducens nerves. Eye 1996; 10: 377-384. 
6 Berlit P. Isolated and combined pareses of cranial nerves III , IV, and VI. A retrospective study of 412 patients. J Neurol Sci 1991; 103: 10-15.

7 Peters GB, Bakri SJ, Krohel GB. Cause and prognosis of nontraumatic sixth nerve palsies in young adults. Ophthalmology 2002; 109: 1925-1928.

8 Moster ML, Savino PJ, Sergott RC, Bosley TM, Schatz NJ. Isolated sixth-nerve palsies in younger adults. Arch Ophthalmol 1984; 102: 1328-1330.
9 Mutyala S, Holmes JM, Hodge DO, Younge BR Spontaneous recovery rate in traumatic sixth-nerve palsy. Am J Ophthalmol 1996; 122: 898-899.

10 King AJ, Stacey E, Stephenson G, Trimble RB. Spontaneous recovery rates for unilateral sixth nerve palsies. Eye 1995; 9: 476-478.

11 Holmes JM, Leske DA, Christiansen SP. Initial treatment outcomes in chronic sixth nerve palsy. J AAPOS 2001; 5: 370-376. 